\title{
Socialisation du groupe-classe au temps de la distanciation sociale
}

\author{
Marion Tellier \\ Université Aix Marseille (France)
}

Group and class socialization in a time of social distancing

doi: $10.18162 / f p .2020 .681$

\section{ésumé}

Pour maintenir l'attention des apprenants dans une classe virtuelle, la dynamique de groupe est une composante essentielle. Cet article rend compte de deux expériences pédagogiques à distance durant la crise pandémique de 2020 : une transposition à distance d'un cours ayant démarré en présentiel avec un groupe se connaissant déjà et la conception d'un module inédit pour la distance. Dans ces deux contextes, la réussite des interactions en ligne et la bonne cohésion groupale ont pu se faire grâce à 3 techniques pédagogiques issues de la dynamique de groupe: faire connaissance, maintenir l'esprit du groupe et participer/collaborer en groupe.

\section{Mots-clés}

Dynamique de groupe, classe virtuelle, transposition, interaction en ligne, cohésion

Abstract

Positive group dynamics are essential for maintaining learner attention in virtual classrooms. This article reports on two remote learning experiments during the 2020 pandemic: remote delivery of a course that was initially held in person with a group that knew each other, and the design of an innovative remote learning module. In both cases, successful online interactions and group cohesion were achieved thanks to three pedagogical techniques based on group dynamics: getting acquainted, maintaining a group spirit, and group participation and collaboration.

Keywords

Group dynamics, virtual classroom, transposition, online interaction, cohesion

\section{Introduction}

En ce printemps 2020, un grand nombre d'enseignants dans le monde ont soudain et brutalement découvert l'enseignement à distance. Si celui-ci existe depuis longtemps, il reste l'apanage de quelques enseignants dans des services spécialisés à l'école primaire, secondaire ou dans le supérieur. En quelques jours, la grande majorité des enseignants ont dû transposer leurs cours en mode distanciel et apprendre à maîtriser des outils et des techniques pédagogiques nouvelles avec plus ou moins de réussite. Un constat que beaucoup d'enseignants ont fait est la difficulté de maintenir l'attention des élèves lors d'un cours en visioconférence. Lorsque l'on est « à l'abri » derrière son écran, il est plus facile de décrocher et de faire autre chose. On perd très vite le fil du cours qu'on était en train de suivre. Certains étudiants ont même déserté les cours. Dès lors, maintenir l'attention des participants lors d'un cours virtuel apparaît comme un enjeu majeur. Cet article se basera sur les analyses d'enregistrements de cours que j'ai dispensés pendant la crise sanitaire. En tant que formatrice de futurs enseignants, j'ai mis en œuvre des techniques que mes étudiants pourront réutiliser dans leur métier. J'étais moi-même relativement peu expérimentée dans la formation à distance au début de cette crise. Cet article se focalisera sur la dynamique de groupe lors d'interactions synchrones en ligne et sur la façon de solliciter l'attention et la participation d'un maximum d'apprenants en stimulant leur implication dans le groupe. J'aimerais ici témoigner de ma pratique et réfléchir à l'impact que cette expérience aura sur mes futurs enseignements, en présentiel comme à distance. En tant que formatrice d'enseignants de langues, une grande part de mes références s'inscrit dans ce champ, mais mon propos dépasse largement la didactique des langues. Après un cadrage théorique sur la dynamique de groupe en présentiel 
comme à distance, je présenterai la méthodologie de ma recherche puis les analyses obtenues suivant trois thématiques : faire connaissance, maintenir l'esprit du groupe et participer/collaborer en groupe.

\section{Dynamique de groupe}

\section{Qu'est-ce qu'un groupe?}

Dans leur ouvrage fondateur, Anzieu et Martin (1968/2007) classifient les groupes humains selon leur structuration, la durée de vie du groupe, le nombre d'individus qui le composent, les relations entre eux, l'effet du groupe sur les croyances et les normes, la conscience des buts à atteindre et les actions communes du groupe. Ils définissent ainsi 5 types : la foule, la bande, le groupement, le groupe primaire ou restreint et le groupe secondaire ou organisation. On peut considérer que le groupe-classe est un groupe primaire ou restreint dans la mesure où son degré d'organisation interne est élevé et la différenciation des rôles est importante. La durée de vie des groupes restreints va de trois jours à dix ans, les relations entre individus étant considérées comme des "relations humaines riches ", génératrices de changement et dont les actions communes des membres du groupe sont qualifiées d'" importantes, spontanées voire novatrices » (1968/2007 :42). Le groupe présente donc un fort potentiel pour générer des actions communes, notamment dans le domaine des apprentissages.

\section{De la nécessité du groupe et des relations sociales dans les apprentissages}

Le lien entre motivation et dynamique de groupe a été mis au jour à la fin du XXe siècle grâce aux travaux de Dörnyei et ses collègues autour de l'apprentissage des langues étrangères (Dörnyei et Malderez, 1997; Dörnyei et Murphey, 2003 entre autres). Le travail de groupe rend possible la mise en place de stratégies collectives, inaccessibles aux individus isolés, et permet à chacun de bénéficier des connaissances et des compétences de chaque membre (Ben Maad et Saadi, 2020). Plusieurs travaux récents ont montré l'influence positive du travail en groupe sur l'apprentissage, la motivation et l'autonomie des apprenants (Ben Maad et Saadi, 2020; Chang, 2010). Cependant, Chang (2010), comme Dörnyei et Murphey (2003) avant elle, souligne l'importance d'être dans un groupe qui fonctionne et qui présente une certaine cohésion, sinon on observe le désengagement des participants et l'effet inverse. La cohésion du groupe s'avère donc fondamentale (Chang, 2010; Dörnyei et Murphey 2003). Elle sert de bouclier social contre la pression des pairs et ses effets potentiellement négatifs, elle permet de réduire le niveau d'anxiété des apprenants et les encourage à maximiser leur effort dans une tâche donnée (Ben Maad et Saadi, 2020). Comme le rappelle Lefranc (2004), les émotions positives comme négatives ressenties par les apprenants ont des impacts sur les apprentissages. Cela est d'autant plus vrai en langue étrangère, où l'insécurité linguistique est forte et où, « explicitement ou implicitement, les énoncés produits en classe sont constamment jugés, et leurs auteurs avec » (Lefranc, 2004 : 84). La cohésion de groupe se manifeste en outre par une implication des apprenants dans leur apprentissage et par un encouragement mutuel entre pairs. Dans la création de cet environnement d'apprentissage positif, le rôle de l'enseignant est fondamental (Chang; 2010, Dörnyei et Malderez 1997; Dörnyei et Murphey, 2003). Il permet aux apprenants de naviguer à travers les différentes étapes de la vie du groupe (Dörnyei et Murphey, 2003), de créer et renforcer les liens et d'éviter les écueils et les conflits.

2 - Formation et profession 28(4 hors-série), 2020 


\section{Comment développer la dynamique de groupe?}

Dörnyei et Malderez (1997) proposent quelques pistes pédagogiques pour renforcer la cohésion entre les membres d'un groupe-classe. Je garderai ici celles sur lesquelles je m'appuie en général dans mes cours en présentiel. Premièrement, il est indispensable de favoriser la formation du groupe en proposant des activités brise-glace pour faire connaissance, partager des informations personnelles et se trouver des points communs (Chang, 2010; Dörnyei et Malderez 1997; Dörnyei et Murphey, 2003). Ces activités mises en place dès la première séance accélèrent le processus de formation du groupe et détendent l'atmosphère. Il est conseillé de réactiver cet état d'esprit régulièrement en démarrant la séance par des activités d'échauffement (Dörnyei et Malderez, 1997). Les mêmes auteurs encouragent les enseignants à multiplier les interactions entre apprenants à travers des travaux en binômes ou en équipes et à privilégier des activités collaboratives plutôt que compétitives (sans pour autant renoncer à une compétition ludique et stimulante). Ces activités collaboratives doivent permettre à chacun d'apporter sa contribution et de se sentir valorisé dans le groupe. La bonne réussite de ce type d'activité repose en grande partie sur l'entente préalable des membres, d'où l'intérêt d'avoir veillé à une formation du groupe stimulante et bienveillante. Dörnyei et Malderez (1997) insistent également sur l'environnement physique d'apprentissage. Il doit permettre aux apprenants de se voir, se rencontrer en se déplaçant dans la salle, voire d'entrer en contact physique et changer de configuration facilement (classe entière, petites équipes, dyades).

La question se pose alors de savoir comment appliquer ces principes et créer la cohésion entre des personnes physiquement distantes et seules face à leur écran.

\section{Et à distance?}

Le passage à l'enseignement à distance trouble nécessairement les repères des apprenants et des enseignants habitués au présentiel. Ne pas se voir (dans les formations asynchrones) ou se voir en mode parfois dégradé (dans de petites fenêtres vidéo dont le flux peut être perturbé), la distance physique, l'immobilité des participants et les difficultés à interagir dans une classe à effectif important peuvent mettre à mal la dynamique de groupe. Pourtant, tout comme en présentiel, « [d]ans les environnements virtuels, le groupe [...] forme un collectif intelligent» (Henri et Lundgren-Cayrol, 2001 : 135). Différents chercheurs ont observé les mécanismes de constitution de communauté d'apprenants en ligne, notamment dans des formations asynchrones (via forum par exemple). Marcoccia (2001) établit 9 conditions à remplir pour constituer une communauté de parole en ligne parmi lesquelles on retrouve le sentiment d'appartenance au groupe et l'émergence d'une histoire commune. Dans une formation asynchrone, on trouve souvent sur un forum, une rubrique « Qui est qui » ou « Faisons connaissance » dont l'objectif principal est de permettre au groupe de construire sa propre identité (Combe Célik 2010 : 289). L'étude de Combe Celik (2010) montre que ce type de rubrique est jugé très utile par les participants et est extrêmement suivi. Les apprenants qui ne se connaissent pas prennent plaisir à se rencontrer virtuellement et à former une communauté de travail. 
L'avènement, dans l'enseignement à distance, d'outils comme la webcam et donc la visioconférence ont permis le développement de formations synchrones. La visioconférence renforce le sentiment de co-présence (Guichon, 2017) et présente de multiples avantages du point de vue psycho-affectif (Develotte et al., 2008). Le fait de pouvoir se voir et échanger en direct vocalement ou via texte (clavardage) est extrêmement pertinent pour la dynamique de groupe. Cependant, il appartient aux enseignants comme aux apprenants d'explorer et de s'approprier les outils des classes virtuelles, car l'utilisation d'une technologie dont on ne maîtrise pas les avantages n'a pas vraiment d'intérêt. Il est essentiel de s'interroger sur ce que cette technologie apporte de neuf ou de mieux pour s'en servir (Walckiers et De Praetere, 2004). L'orchestration des modalités offertes par l'outil est nécessaire pour gérer les différents aspects de l'interaction didactique (Guichon et Tellier, 2017).

\section{Recueil de données}

Les données utilisées ici proviennent de deux sources. Il s'agit tout d'abord d'enregistrements vidéo de 6 séances de cours à distance d'environ $1 \mathrm{~h}$ chacune. Tous les cours ont eu lieu dans le cadre de la formation en $1^{\text {re }}$ année de master Didactique du FLE en situation de plurilinguisme à Aix Marseille Université (France) où les étudiants suivent des cours de didactique du FLE afin de devenir enseignants. Ils sont habitués à utiliser la plateforme pédagogique de l'université et lors du $2^{\mathrm{e}}$ semestre : avant que le gouvernement français n’impose la fermeture des établissements scolaires et un confinement de la population, nos étudiants suivaient un cours dédié à l'enseignement en ligne "Concevoir et tutorer des cours de langue en ligne ». Dans ce module, ils apprenaient en outre à utiliser la plateforme de classe virtuelle Amuconnect. La promotion 2019-2020 comptait 24 étudiants et lors des séances à distance, ils étaient entre 19 et 22. L'enseignante ${ }^{1}$, c'est-à-dire moi-même, possédant 20 ans d'expérience pédagogique, en particulier dans la formation d'enseignants de FLE, est spécialisée dans la dynamique de groupe. ${ }^{2}$ J'enseigne très ponctuellement à distance, notamment dans un séminaire synchrone de master 2 co-animé avec des collègues sur la plateforme Amuconnect, dérivée d'Adobe connect et acquise par notre université. Au moment du confinement, j'avais une très faible maîtrise de cette plateforme de classe virtuelle et je n'avais d'ailleurs jamais créé moi-même de session. Elle permet, comme beaucoup de logiciels de classe virtuelle, plusieurs vues (présentation, discussion, collaboration) et donne également la possibilité de travailler en sous-groupes (ateliers). Amuconnect permet en outre d'enregistrer la séance, ce que j'ai fait systématiquement pour permettre aux étudiants absents pendant les séances synchrones de les visualiser. Ces séances n'ont pas été enregistrées à des fins de recherche et l'ensemble constitue donc un corpus écologique. Il est composé de 4 séances issues du cours « Enseigner les langues aux enfants » et d'un « café virtuel » organisé avec les étudiants après la fin des cours pour garder le contact alors nous étions encore confinés. ${ }^{3}$ Les extraits du corpus seront codés M1-enf (cours sur les enfants) et M1-café (café virtuel).

À cela s'ajoutent des documents issus d'une formation continue en ligne dans une université d'été (SUPFLES) pour enseignants de FLE dans laquelle je suis intervenue au début du mois de juillet 2020 pour un module de $2 \mathrm{~h}^{4}$ sur les activités favorisant la dynamique de groupe en présentiel et à distance. Il y avait 8 participantes. Lors de ce module, j’ai commencé à formaliser la pratique pédagogique expérimentée pendant nos cours confinés de master 1 et que je développerai dans cet article. Les extraits de ce module seront codés Mod-2020.

4 - Formation et profession 28(4 hors-série), 2020 
En plus de toutes ces séances de classe virtuelles, j'utiliserai également des extraits de courriels envoyés aux participants avant ou après les différentes séances (master 1 ou module de formation continue).

\section{Analyse des données}

Trois aspects qui me semblent importants pour rendre les cours en classe virtuelle dynamiques seront développés ici : 1) Faire connaissance, 2) Développer l'esprit de groupe et 3) Participer et collaborer en groupe.

\section{Faire connaissance}

Nous avons évoqué dans la partie théorique la nécessité pour l'enseignant d'établir une ambiance bienveillante, notamment lors de la formation du groupe (Chang, 2010; Dörnyei et Murphey, 2003) afin de faciliter ensuite le travail collaboratif. Le gros avantage de la distance est que lorsque l'on apparaît avec notre image à l'écran, notre nom s'affiche en dessous et cela facilite grandement la mémorisation des prénoms de chacun (voir figure 3 pour un exemple). On peut ainsi se focaliser plutôt sur l'échange d'informations visant à mieux faire connaissance. Dans le module de formation continue, les participants ne se connaissaient pas, alors j'avais envoyé des consignes par courriel avant la séance pour que chacun crée son blason (figure 1).

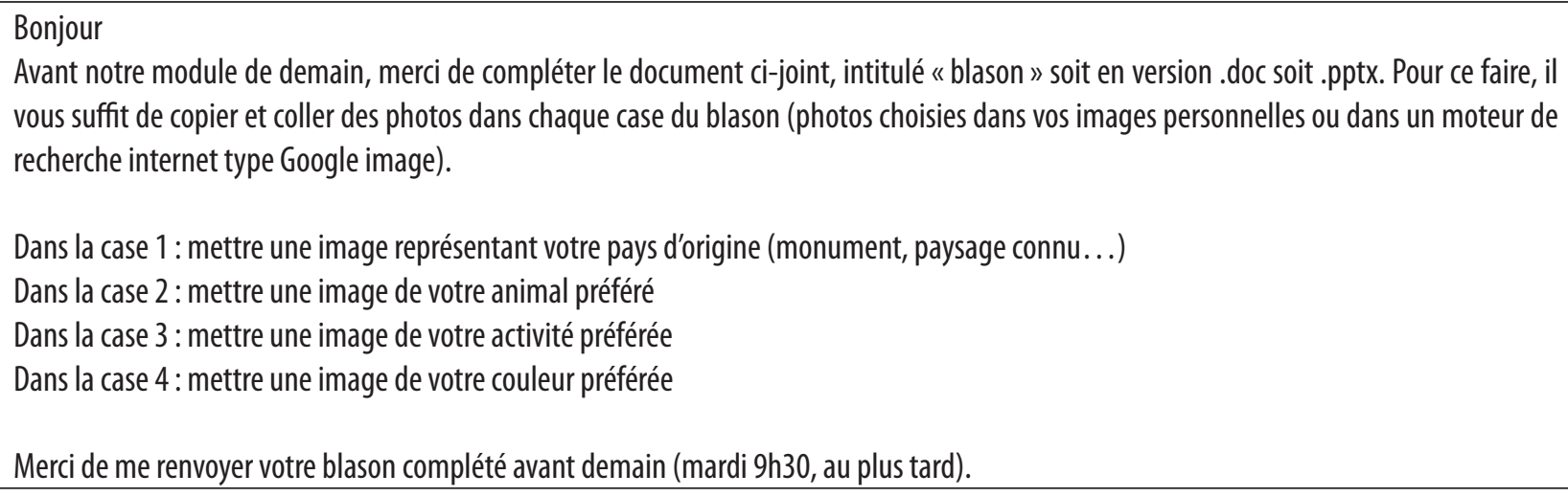

Figure 1

Courriel de consigne pour le blason (Corpus Mod-2020)

J'ai ensuite compilé les blasons (exemples en figure 2) et nous nous sommes posé des questions afin de deviner à qui chaque blason appartenait. Cela nous a permis de mieux faire connaissance et a donné à chacun la possibilité de prendre la parole et d'interroger les autres. 

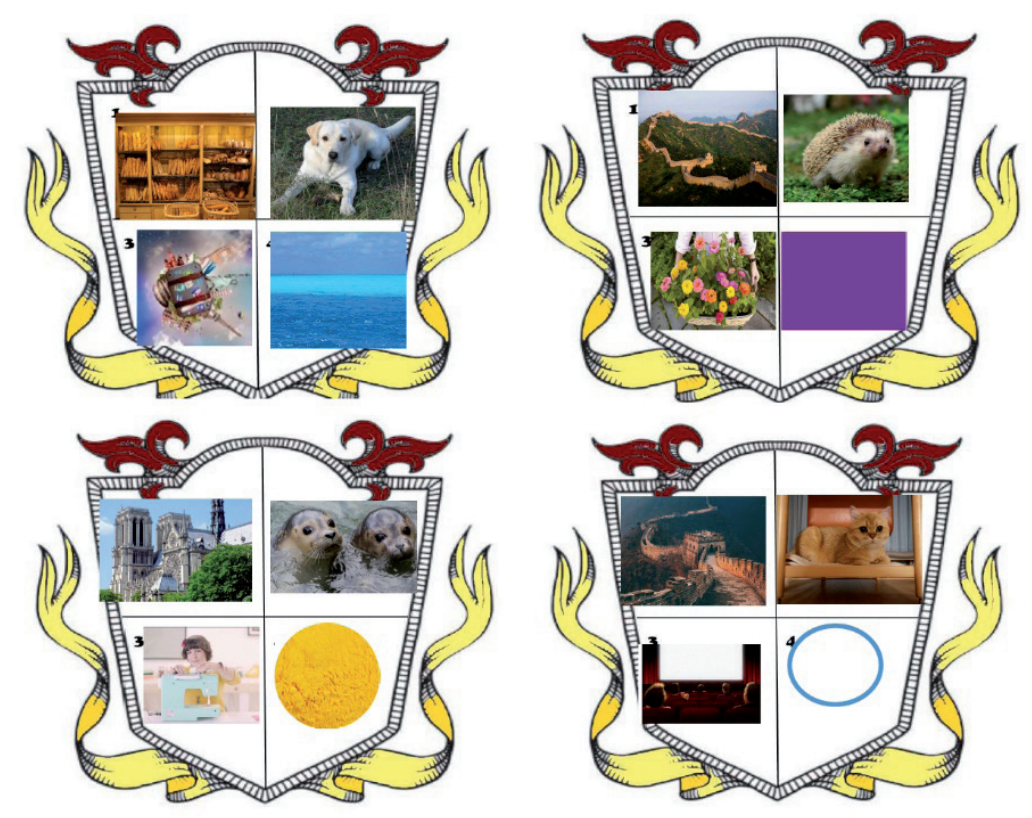

Figure 2

Exemples de blasons des participants (Corpus Mod-2020)

\section{Développer l'esprit de groupe}

Dörnyei et Malderez nous mettent en garde (1997) : briser la glace ne suffit pas, il faut constamment réchauffer le climat. Ainsi, sans alimentation de la cohésion de groupe, même un groupe sain et dynamique peut péricliter (Dörnyei et Murphey, 2003). Un groupe-classe est un microcosme avec son propre système de règles, sa culture et son histoire (Henri et Lundgren-Cayrol, 2001) et il convient de maintenir cet état d'esprit et de renforcer le sentiment d'appartenance au groupe. J'ai testé deux approches dans ce sens : faire des « photos de classe » et mettre en place des moments conviviaux.

\section{Photo de classe}

Contrairement au présentiel, quand on est à distance et que chacun affiche sa vidéo, on peut voir d'un seul coup les visages de tout le monde. Pourquoi ne pas en profiter pour faire une capture d'écran et saisir cet instant comme une photo de classe ? On peut s'amuser à prendre des poses et même ajouter une petite pincée d'humour.

Cette illustration vient renforcer l'esprit de groupe et peut être un souvenir que l'on garde comme une vraie photo de classe (figure 3). Ces photos sont peu coûteuses en temps de classe et ne requièrent pas de maîtrise technologique complexe. 


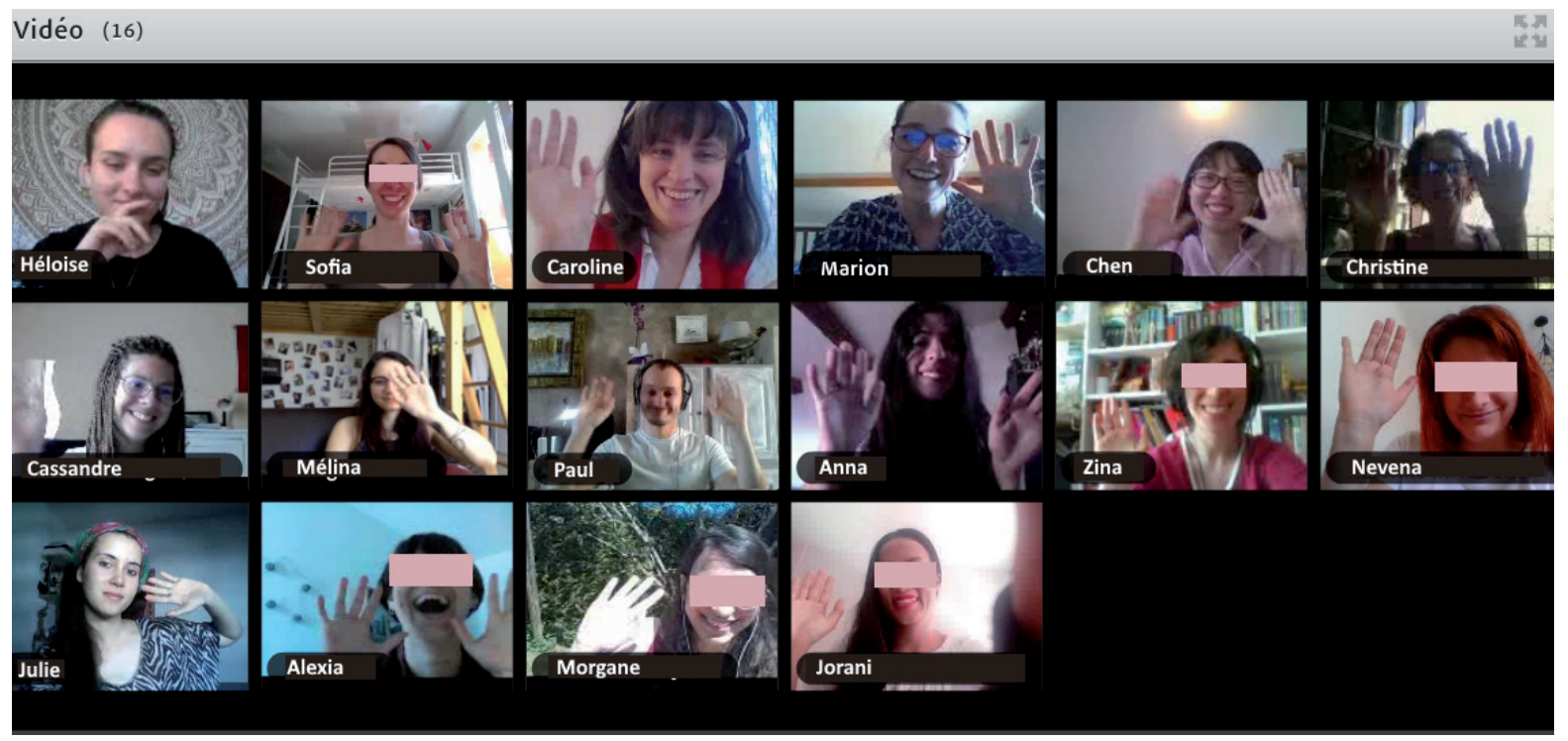

Figure 3

Photo de classe en mode distanciel (Corpus M1-enf)

Une autre photo de classe que nous avons faite était directement liée au contenu pédagogique. Dans un autre cours de master 1 sur les techniques de créativité en classe de langue, nous avions le projet de réaliser une œuvre d'art commune sur le thème « joie et bonheur ». Nous devions chacun produire quelque chose (dessin, peinture, collage, pliage...) sur ce thème et ensuite coller tout ensemble sur un grand panneau. Malheureusement, le confinement nous a empêchés de le faire. Le cours étant passé à distance et animé par une autre collègue pour les séances suivantes, celle-ci et les étudiants ont eu l'idée de faire ce collage d'œuvres en mode numérique, chacun faisant apparaître sa production à l'écran le temps d'une capture (figure 4).

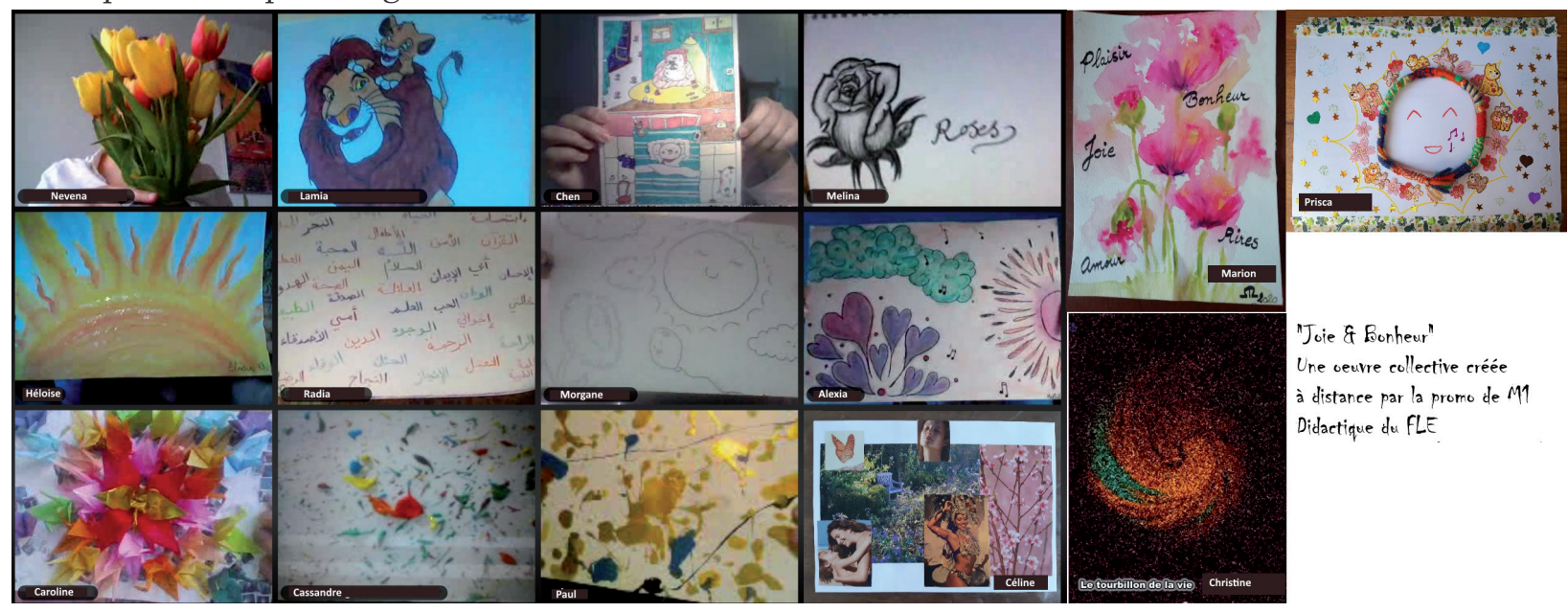

Figure 4

L'euvre d'art collective "Joie et Bonheur" 
La constitution de cette œuvre collective a été un moment fort dans notre groupe, le thème de la joie et du bonheur étant tout particulièrement le bienvenu dans cette période de confinement. La photo de l'œuvre a ensuite été utilisée sur la page d'accueil Facebook de notre département universitaire. Cet évènement a contribué à l'histoire partagée du groupe et au renforcement de ses liens (Dörnyei et Malderez, 1997).

\section{Les moments conviviaux}

Chang (2010) nous invite à organiser des moments conviviaux pour souder le groupe, envisageant même des regroupements autour de quelques boissons et nourritures à partager. Au temps de la distanciation sociale, même en présentiel, ce genre d'initiative est impossible. Quelles alternatives s'offrent à la formation à distance ? En mode asynchrone, on trouve souvent sur les forums un espace informel. Combe Celik a étudié les messages échangés dans la rubrique « Récré » d'un forum. Elle note le caractère récréatif des messages et leur « fonction essentiellement socio-affective » $(2010: 153)$ dans lesquels sétablit un mode de communication informel parfois très personnel.

En mode synchrone, ces échanges informels apparaissent souvent en début de séance au moment de l'accueil des participants. L'extrait de corpus suivant (figure 5) en est un exemple. ${ }^{5}$

\begin{tabular}{|c|c|}
\hline 1 & MT : alors je vois pas tout le monde là //y en a qui veulent pas se montrer je suis sûre qu'il y en a \\
\hline 2 & qui sont en pyjama \\
\hline 3 & [Lamia : non mdr] \\
\hline 4 & [Anna : je vous assure que non $\mathbf{m d r}$ ] \\
\hline & MT rit en lisant le clavardage \\
\hline 5 & [Paul: non haha] \\
\hline 6 & MT : OK / bon ben écoutez j'espère que vous allez bien / que vous tenez le coup / que tout le \\
\hline 7 & monde est en bonne santé / c'est Jamila qui était malade c'est ça ? // vous avez des nouvelles? \\
\hline 8 & [Christine : Jamila est sortie de l'hopital] \\
\hline 9 & [Leila : elle est sortie de l'hopital mais est toujours malade elle n’a plus de voix] \\
\hline 10 & [Christine : c'est Leila qui a des nouvelles] \\
\hline 11 & [Leila : merci à vous:)] \\
\hline $\begin{array}{l}12 \\
13\end{array}$ & $\begin{array}{l}\text { MT : (lit le clavardage à haute voix) Jamila est sortie de l'hôpital d'accord / donc ça va mieux alors ? elle est toujours } \\
\text { malade et n'a plus de voix peuchère } 0 \mathrm{~K} \text { / merci Leila // vous lui direz }\end{array}$ \\
\hline 14 & qu'on pense bien à elle et qu'on l'embrasse hein \\
\hline
\end{tabular}

\section{Figure 5}

"Je suis sûre qu'il y en a qui sont en pyjama» (corpus M1-enf) 
Dans cet extrait, on note tout d'abord qu'il existe une véritable dynamique conversationnelle multimodale. En effet, si en tant qu'enseignante, je m'exprime uniquement vocalement, les étudiants, eux, n'utilisent que le clavardage afin de participer à cette discussion spontanée sans chevauchement de parole. Plusieurs éléments témoignent du côté informel de la conversation (et d'ailleurs un peu éloigné du style d'interaction habituelle dans le cadre universitaire). J'ouvre la séquence (1.1-2) par une plaisanterie " je suis sûre qu'il y en a qui sont en pyjama ", cette remarque faisant partie de l'histoire conversationnelle du groupe, car avant la toute première séance à distance et alors que le confinement commençait à peine, j’avais envoyé le courriel suivant aux étudiants : "À jeudi et pensez à vous organiser pour maintenir un rythme de travail et ne pas passer la journée en pyjama. Webcam obligatoire jeudi, je vais vérifier!!!! ». Les étudiants réagissent en montrant leur amusement par des marques informelles « $\mathbf{m d r}$ » (1. 3 et 1.4$)$ ou encore «haha» (1. 5). Témoignant toujours du contexte particulier de la pandémie, je poursuis alors sur un ton plus sérieux en m'inquiétant de leur santé tant physique que mentale (1. 6-7), puis en demandant des nouvelles d'une étudiante hospitalisée (Jamila). L'étudiante Christine répond qu'elle est sortie de l'hôpital, puis Leila confirme et développe. Ce qui est intéressant ici, c'est que Leila est très amie avec Jamila et que, comme le confirme Christine (1.10), c'est elle qui a des nouvelles directes. Le fait que Christine ait pourtant répondu en premier montre que le groupe (au moins une partie) avait déjà eu des nouvelles de Jamila. Cette solidarité entre eux est le résultat d'une dynamique déjà bien construite dès le semestre précédent. Ma dernière intervention (1.12-14) demeure dans le registre informel avec l'utilisation du terme " peuchère » (caractéristique du sud de la France et signifiant « la pauvre»). Je demande aussi à Leila de dire à Jamila quon pense bien à elle et qu'on l'embrasse, me faisant ainsi porte-parole du groupe (usage du pronom « on »). Cette intervention témoigne de l'aspect socio-affectif de l'échange.

Outre ces débuts de séance conviviaux, j’ai également organisé 2 cafés virtuels après la période de cours alors que nous étions encore confinés. À cette époque, les étudiants préparaient leurs dossiers à rendre et leurs examens pour valider leur année universitaire. En temps normal, nous marquons la fin de l'année universitaire par un pique-nique. Ne pouvant organiser cet évènement dans le contexte de distanciation sociale et sentant que beaucoup d'étudiants étaient esseulés, voire déprimés, je leur ai proposé un café virtuel. Tous ceux qui le souhaitaient (enseignants comme étudiants) ont participé à ce rendez-vous, munis d'une tasse de thé ou de café (ce qui nous a permis de faire encore une belle photo de groupe).

Sachant que le dispositif de la classe virtuelle rendait difficile la discussion spontanée, j’ai proposé une petite activité de détente, annoncée la veille par le courriel suivant: "pour ceux qui le soubaitent, vous pouvez menvoyer par mail avant $12 \mathrm{~b}$ demain, une photo qui représente votre confinement (un objet, un meuble, un lieu, un animal domestique...) sans que l'on voie de visage. On essaiera de deviner qui a envoyé la photo.». Ces photos (exemples figure 6) étaient montrées une par une. Les étudiants répondaient dans le clavardage en faisant des hypothèses sur leur auteur. Cette activité permettait d'entrer dans l'intimité de chacun (j'ai d'ailleurs moi-même participé) et de renforcer les liens tout en passant un bon moment. 


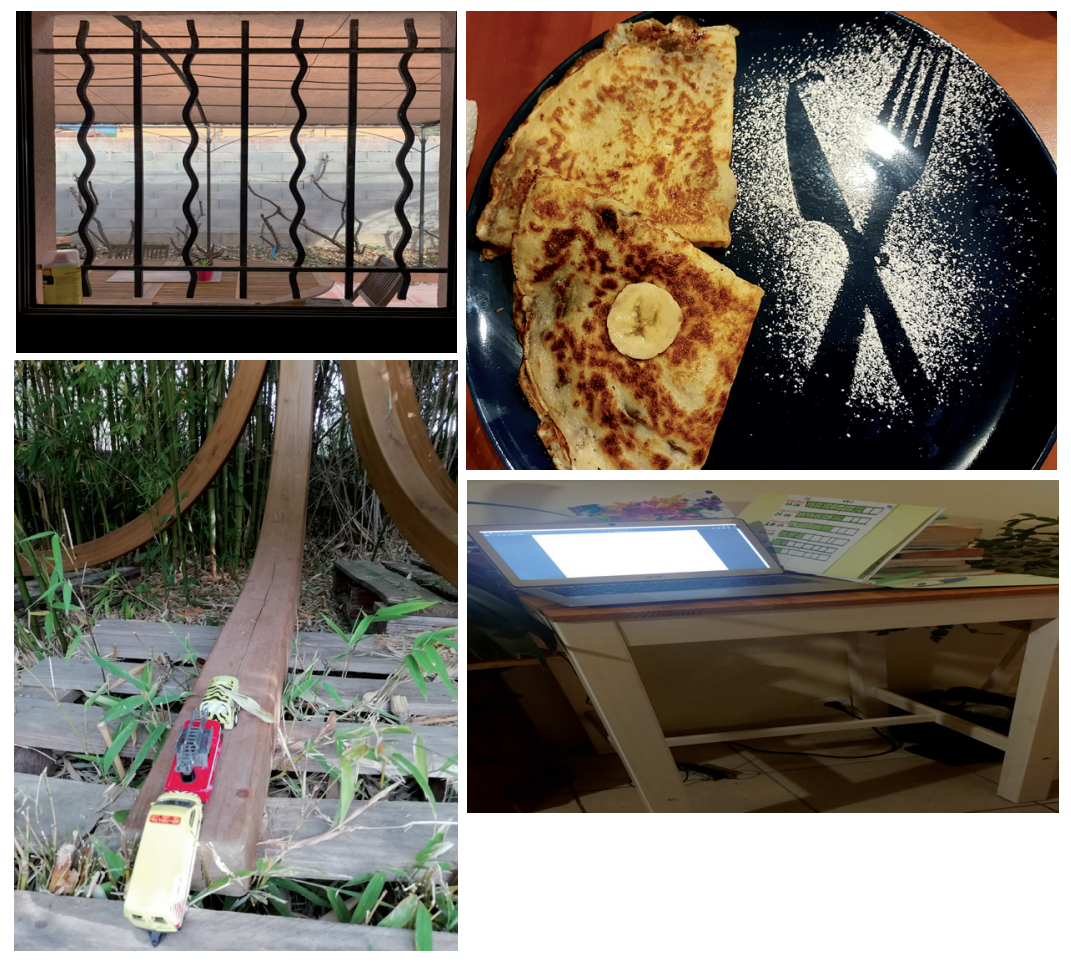

Figure 6

"Maphoto du confinement» (corpus M1-Café)

\section{Participer et collaborer en groupe}

Comme évoqué dans la partie théorique, une bonne entente dans le groupe et une bonne cohésion sont essentielles pour le faire fonctionner. Je voudrais, dans cette dernière partie, aborder la question sur travail en groupe sous deux angles : la transposition à distance et la conception pour la distance.

\section{La transposition}

Prise de court comme beaucoup de collègues, mes premières séances à distance ont été préparées dans l'urgence et reposaient sur la transposition des activités que j'aurais dû faire en présentiel, adaptant les modes de participation en fonction des contraintes de l'outil. Comme évoqué plus haut, l'interaction voco-verbale spontanée n'est pas évidente en visioconférence et si chacun se met à parler en même temps, c'est inaudible. Néanmoins, la participation active des étudiants s'avère essentielle au maintien de leur attention. J'avais prévu un exercice dans le cours sur l'enseignement des langues aux enfants dans lequel chacun devait se prononcer sur différentes propositions pédagogiques (sont-elles adaptées à l'enseignement des langues aux enfants ?). Une fois encore, j'ai demandé aux étudiants de faire l'exercice avant la séance et nous avons corrigé ensemble (figure 7). Notre méthode était la suivante : je lisais chaque proposition à haute voix et les étudiants signalaient si leur réponse était « oui » ou « non » par un geste (pouce levé pour oui, baissé pour non) pour ceux qui étaient en vidéo (Caroline avait même préparé des petits panneaux signalétiques pour répondre). Pour ceux qui ne pouvaient pas 
mettre leur vidéo, ils écrivaient dans le clavardage. Les différentes modalités de la classe virtuelle et leurs affordances sont ici encore bien exploitées et tout le monde reste attentif.

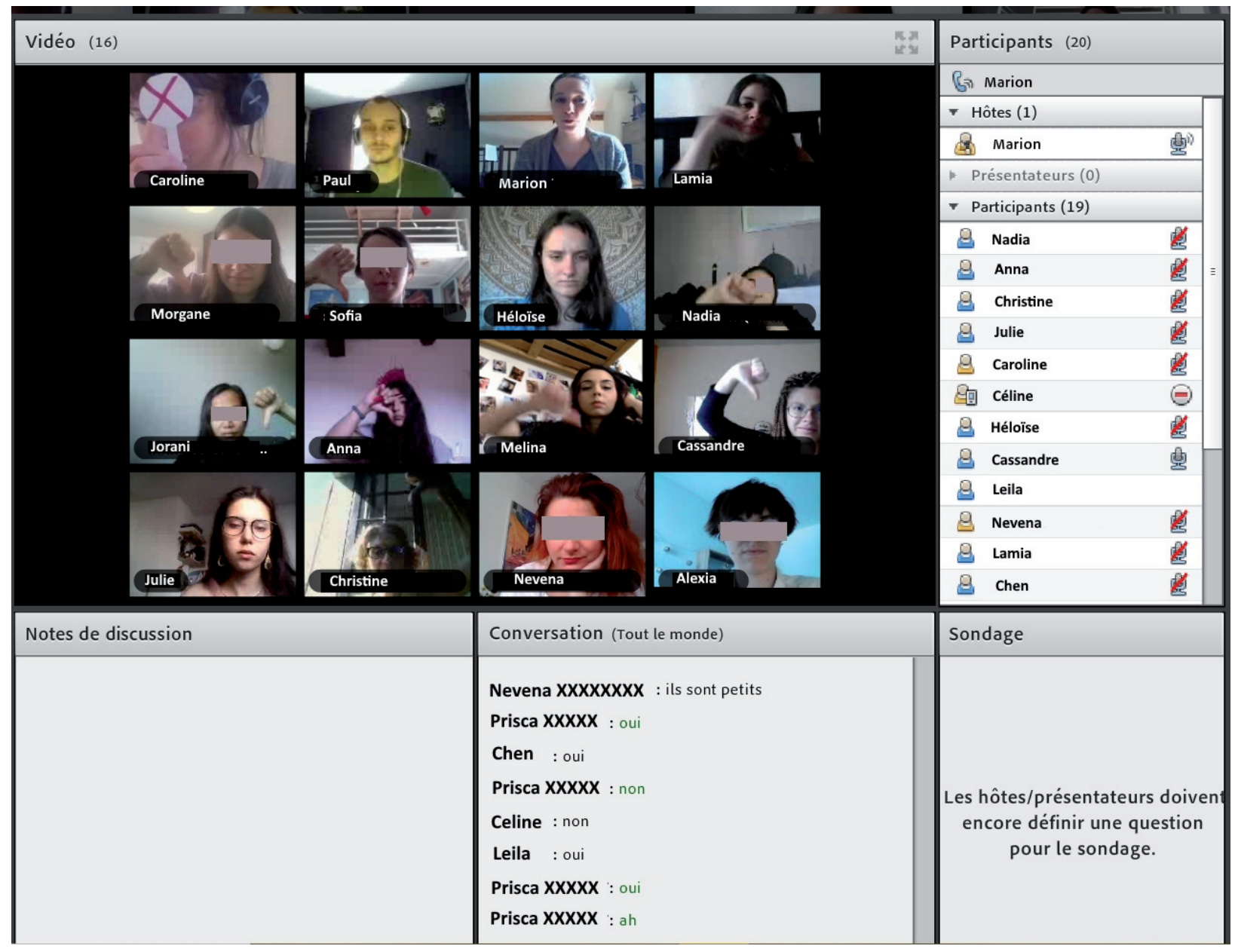

Figure 7

Questions-réponses dans la classe virtuelle (Corpus M1-enf)

Cette transposition ne s'est pas toujours faite aussi facilement. Dans la transcription suivante (figure 8), j'essaie d'adapter une activité d'échauffement que nous avons déjà faite en présentiel (où on se lance des ballons imaginaires en interpellant le destinataire du ballon seulement par contact visuel). Le but est de créer une synergie entre les participants de manière récréative. 


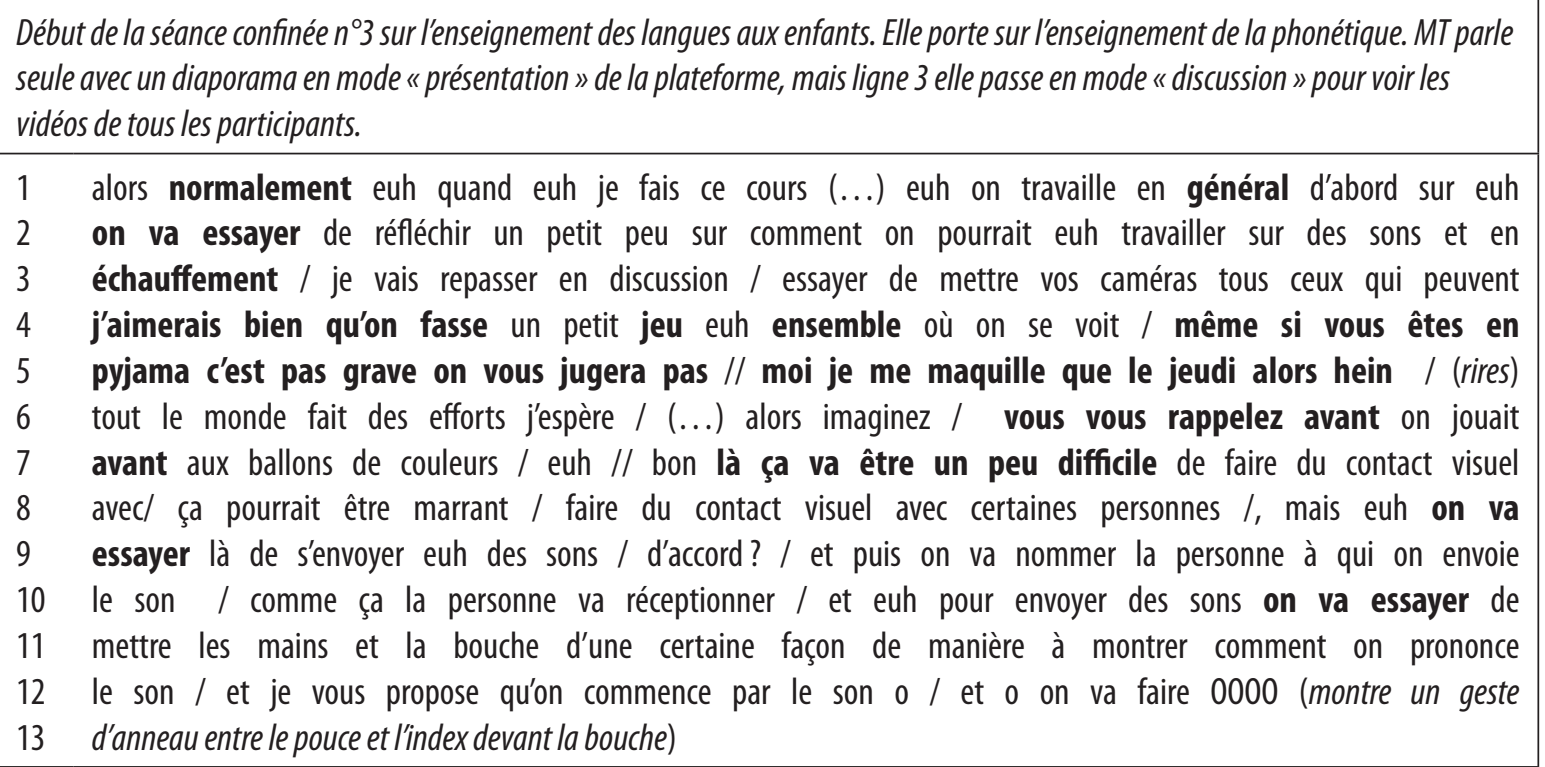

Figure 8

"On va essayer» (Corpus M1-enf)

Même si on retrouve dans mon discours des éléments caractéristiques de la dynamique de groupe : l'activité d' « échauffement" (1. 3), le côté ludique " jeu » «ensemble " (1. 4), la référence à l'histoire commune du groupe : "même si vous êtes en pyjama» (1. 4-5) et enfin la confidence « moi je me maquille que le jeudi alors hein" (1. 5) (le jour du cours), on sent que la situation est singulière et nécessite une transposition. Celle-ci est tout à fait visible dans mon discours, j'emploie des termes comme «normalement» (1.1) et «en général» (1. 1). La situation est donc inédite et vient rompre avec nos habitudes : "vous vous rappelez avant on jouait avant» (1.6). On voit aussi en filigrane une certaine insécurité quant à la réussite de l'activité : « on va essayer » apparaît à 3 reprises $(1.2,8-9$ et 10) ainsi que « là ça va être un peu difficile». Cependant, habitués à ce genre d'exercice, les étudiants ont collaboré et l'activité s'est plutôt bien déroulée.

\section{La conception pour la distance}

Concevoir des activités pédagogiques directement pour le mode distanciel est sans doute plus efficace, d'autant plus si l'enseignant exploite les potentialités des outils numériques. Dans tous les exemples que j'ai donnés ici, les étudiants participaient en classe entière. Cependant, plusieurs dispositifs de classe virtuelle permettent de travailler en sous-groupes. On commence en classe entière, ce que Henri et Lundgren-Cayrol (2001 : 47) appellent le " quartier général », pour donner les consignes. Puis l'enseignant répartit (aléatoirement ou non) les étudiants en petits groupes. Dans la figure 9, une capture d'écran du module de formation continue, on voit, dans la colonne de droite, que les participantes sont réparties en groupes de 4 dit « ateliers ». La capture d'écran nous montre l'intérieur de l'atelier 2. Les participantes ont 5 minutes pour trouver un maximum de femmes françaises célèbres $\mathrm{du} \mathrm{XX}^{\mathrm{e}}$ siècle et feront marquer un point à leur équipe pour chaque réponse correspondant aux 10 
femmes que l'enseignante a secrètement listées. L'effectif étant plus restreint, les participantes peuvent s'exprimer oralement via leur micro et/ou dans le clavardage ("Conversation »), puis elles synthétisent leurs réponses dans la fenêtre « Notes » à gauche. Cette interface permet à l'enseignante ("Marion ») de visiter les différents ateliers et voir comment se déroule l'activité. Dans le processus de remueméninges, le groupe prend toute son importance puisque chacun peut contribuer en apportant ses connaissances et rebondir sur les idées des autres pour en trouver de nouvelles.

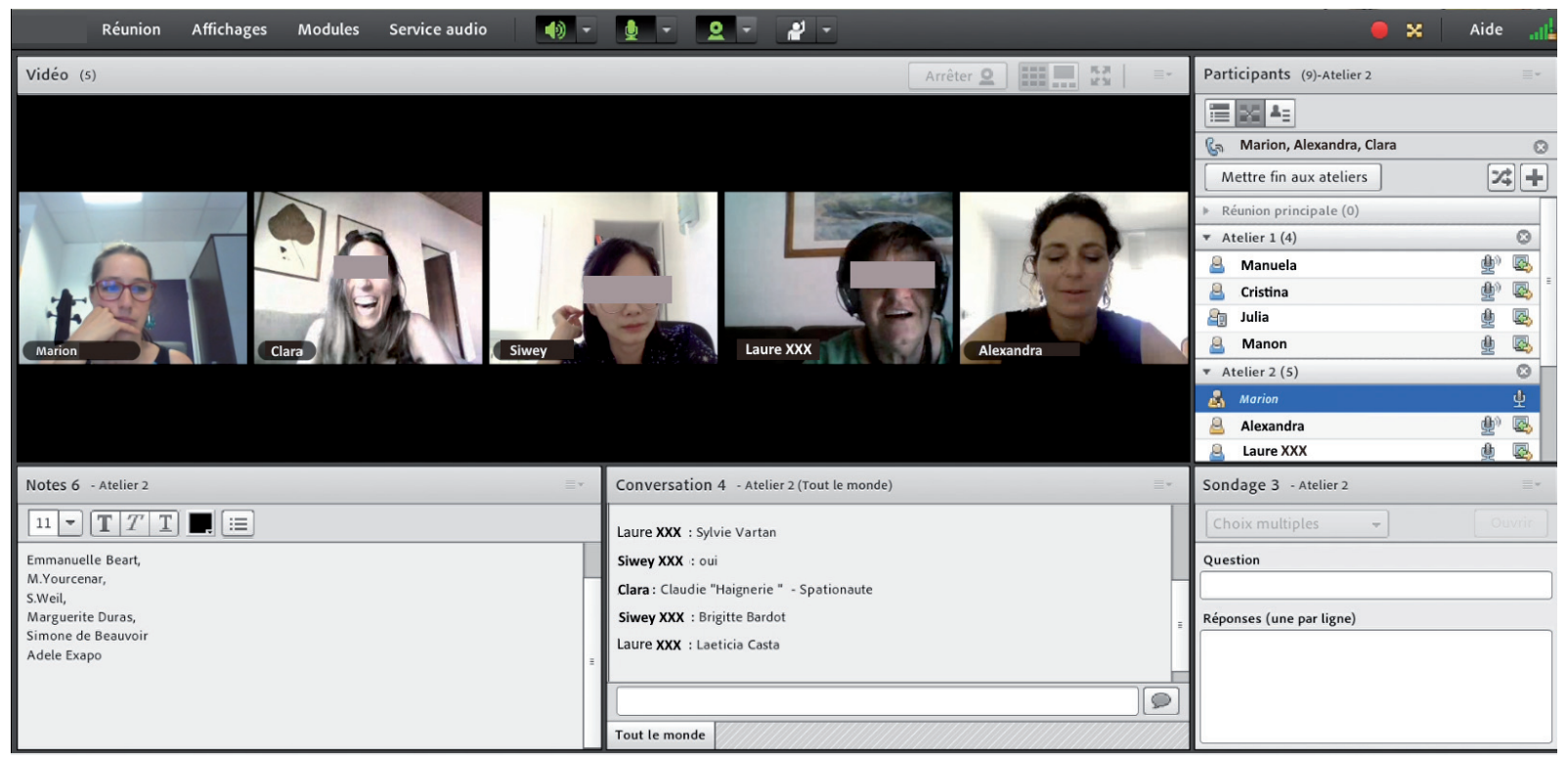

Figure 9

Remue-méninges en ateliers (Corpus Mod-2020)

Une autre activité que j'ai testée dans la même formation était une enquête policière à mener en atelier. Avant la séance en visioconférence, j'avais envoyé par courriel à chaque membre de chacun des 2 ateliers quelques indices pour résoudre cette enquête. Le mystère ne pouvait être résolu qu'en mettant tous les indices en commun. La contribution de chaque membre de l'équipe était donc indispensable à la réussite de l'activité. Je la pratique souvent en présentiel, mais, si elle fonctionne très bien globalement, je remarque que les participants ont tendance à poser tous les indices sur la table (ils les ont sur des petits papiers) et collaborent en ayant tout sous les yeux. À distance, les deux équipes ont fonctionné différemment. Dans l'une, les discussions se sont faites oralement et chacun participait en argumentant avec ses propres indices; dans l'autre, certains participants ont recopié fastidieusement leurs indices dans la partie « Notes ». La première équipe a rapidement et efficacement résolu le mystère, tandis que la seconde n'y est pas arrivée. À la décharge de cette dernière, de nombreux problèmes techniques ont perturbé les échanges oraux des participantes. 


\section{Conclusion}

Pour reprendre les propos d'Henri et Lundgren-Cayrol « la collaboration, surtout en mode virtuel, est (...) un exercice qui s'apprend. Savoir collaborer n'est pas inné: cela s'apprend, au même titre que des connaissances ou des compétences disciplinaires. » 2001 : 152) Pour ce faire, " préparer » le groupe est indispensable : faire connaissance, maintenir l'esprit de groupe et proposer des activités où chacun peut participer est essentiel. Comme beaucoup d'enseignants, j’ai transposé à distance mes cours pensés pour le présentiel. Cela s'est parfois très bien passé, alors qu'à d'autres moments, certaines activités ont été plus laborieuses. Cependant, cette expérience m'a permis de développer mes compétences technosémio-pédagogiques, c'est-à-dire ma « capacité à utiliser les outils de communication à disposition et les modalités (texte, son, image, temps) qui leur sont propres de manière opportune pour l'apprentissage de la L2.»(Guichon, 2011 : 100) Si ce concept a été développé pour l'enseignement des langues étrangères, on voit qu'il s'adapte à tout enseignement. Ainsi, lorsque j'ai dû, peu de temps après, créer un petit module de formation continue à distance, il a été plus aisé de penser directement les activités avec les contraintes, mais aussi les potentialités de la classe virtuelle.

Alors qu'aucun parmi nous ne peut prédire dans quel contexte aura lieu la prochaine rentrée scolaire, j'envisage déjà la refonte de mes cours de master pour la distance. Un des principaux cours que je donne portant sur la dynamique de groupe, les principes listés dans cet article seront essentiels. Il me semble aussi que déléguer une partie de l'activité en amont permet de gagner du temps. Dans cet article, on a vu à plusieurs reprises que j'avais envoyé par courriel des consignes aux participants avant la séance afin qu'ils préparent certaines choses.

Les activités présentées ici fonctionnent très bien dans un monde idéal, mais il faut bien admettre que la réalité de la classe virtuelle est tout autre. Il y a une grande part d'imprévisibilité et de dysfonctionnements bien plus fréquents qu'en présentiel. La gestion des problèmes matériels divers (micro ou webcam, connexion internet) perturbe nécessairement le bon déroulement des séances et rend les interactions difficiles, voire pénibles. En effet, les problèmes de chevauchements, les interruptions de l'échange et la disparition d'une ou plusieurs modalités peuvent mettre en péril la planification de l'enseignant (Azaoui, 2017). Il faut donc faire preuve de « flexibilité pédagogique » et se constituer un « répertoire d'actions de rechange » (Develotte et al., 2008, paragr. 80) en cas de perturbation. Cela implique donc que la planification de cours à distance envisage différents scénarios de secours pour maintenir les échanges et la dynamique de groupe coûte que coûte.

\section{Notes}

1 L'auteure de la publication est également l'enseignante du corpus, j'ai décidé d'écrire cet article à la première personne du singulier pour ainsi mieux restituer mon point de vue d'enseignante-chercheure.

2 Pour voir un exemple de ma pratique pédagogique en présentiel, voir ici : https://amupod.univ-amu.fr/video/1628-1pedagogie-favoriser-les-relations-sociales-dans-le-groupe/

3 Lorsqu'il s'est agi de mener une recherche sur ces enregistrements, les étudiants ont été contactés afin de signer un formulaire de consentement pour autoriser l'utilisation des vidéos. Les visages des étudiants n'ayant pas répondu ont été masqués. Tous les noms ont été effacés ou modifiés dans les transcriptions.

4 Intitulé du module: «Quelles pratiques de classe pour stimuler la dynamique de groupe et l'oral ? Quelles transpositions pédagogiques pour l'enseignement en ligne? » https://www.univ-amu.fr/system/files/2020-07/Brochure\%20SUPFLES univ \%20e\%CC\%81te\%CC\%81\%202020 Distance diffusion.pdf 
5 Les transcriptions correspondent aux échanges en temps réel. Les parties entre crochets et sur fond gris sont écrites dans la partie clavardage. Les parties en italique sont des commentaires que j'ajoute. Les parties en gras sont mises en évidence pour l'analyse de cet extrait. / correspond à une pause courte, // à une pause longue.

\section{Références}

Anzieu D. et Martin J.-Y. (2007). La dynamique des groupes restreints. PUF. (Ed. orig. 1968).

Azaoui, A. (2017). Faire face aux imprévus techniques. Dans N. Guichon et M. Tellier (dir.), Enseigner l’oral en ligne - une perspective multimodale (p.116-134). Éditions Didier.

Ben Maad, MR, Saadi, I. (2020). The role of group dynamics in low-achieving EFL students' speaking development. International Journal of Applied Linguistics, 30(2), 1-16. https://doi.org/10.1111/ijal.12279

Chang, L.Y. H. (2010). Group Processes and EFL Learners' Motivation: A Study of Group Dynamics in EFL Classrooms. TESOL Quarterly, 44(1), 129-154. https://doi.org/10.5054/tq.2010.213780

Combe Celik, C. (2010). Pratiques discursives dans une formation en ligne à la didactique du français langue étrangère : une analyse de la communication pédagogique asynchrone [Thèse de doctorat non publiée]. Université Stendhal - Grenoble III. Repérée le 21 juillet 2020 à https://tel.archives-ouvertes.fr/tel-00508363

Develotte, C., Guichon, N. et Kern, R. (2008). «Allo Berkeley ? Ici Lyon... Vous nous voyez bien ? » Étude d'un dispositif de formation en ligne synchrone franco-américain à travers les discours de ses usagers. Alsic, 11(2). Repéré le 21 juillet 2020 à http://journals.openedition.org/alsic/892; https://doi.org/10.4000/alsic.892

Dörnyei, Z., et Malderez, A. (1997). Group dynamics and foreign language teaching. System, 25(1), 65-81. https://doi.org/10.1016/S0346-251X(96)00061-9

Dörnyei, Z., et Murphey, T. (2003). Group dynamics in the language classroom. Cambridge University Press. https://doi.org/10.1017/CBO9780511667138

Guichon, N. (2017). Se construire une présence pédagogique en ligne. Dans N. Guichon et M. Tellier (dir.), Enseigner l'oral en ligne - Une approche multimodale (pp. 29-58). Éd. Didier.

Guichon, N. \& Tellier, M. (2017). (dir.) Enseigner à l’oral en ligne : une approche multimodale des interactions pédagogiques (pp. 59-90). Paris : Didier.

Henri, F. et Lundgren-Cayrol, K. (2001). Apprentissage collaboratif à distance. Pour comprendre et concevoir les environnements d'apprentissage virtuels. PUQ. Repéré le 21 juillet 2020 à https://www.puq.ca/catalogue/livres/apprentissage-collaboratif-distance-92.html

Lefranc, Y. (2004). FLE, FL«M», FLS : les apprenants, leur faculté de langage et la classe de langue. Éla. Études de linguistique appliquée, 133(1), 79-95. doi:10.3917/ela.133.0079 .

Marcoccia, M. (2001). L'animation d'un espace numérique de discussion : l'exemple des forums usenet. Document numérique, 5(3), 11-26. doi:10.3166/dn.5.3-4.11-26.

Walckiers, M. et De Praetere, T. (2004). L'apprentissage collaboratif en ligne, huit avantages qui en font un must. Distances et savoirs, 2(1), 53-75. doi:10.3166/ds.2.53-75.

\section{Pour citer cet article}

Tellier, M. (2020). Socialisation du groupe-classe au temps de la distanciation sociale. Formation et profession, 28 (4 hors-série), 1-15. http://dx.doi.org/10.18162/fp.2020.681 